\title{
EDUCAÇÃO EM DIABETES: DO ACOLHIMENTO AO AUTOCUIDADO
}

\author{
Gleicy Kelle Alves Damas ${ }^{1}$, Bruna de Paula Santana², Thaís Ma- \\ rielly de Souza Pires 3 , Amanda dos Anjos Brandão3 ${ }^{3}$, Maria Clara \\ D'Alcântara Pereira ${ }^{3}$, Vivia Ribeiro Castro ${ }^{3}$, Ana Luiza Araújo da \\ Silva ${ }^{3}$, Danielly Rezende LourençO ${ }^{3}$, Rosana de Morais Borges Mar- \\ QUeS $^{4}$, Maria Luiza Ferreira Stringhini ${ }^{4}$
}

\begin{abstract}
Resumo: A educação em diabetes é um processo contínuo de acesso ao conhecimento para que pacientes desenvolvam habilidadesparaogerenciamentoda doença.Pensando assim, o presente projeto ocupa o tempo de espera por consultas hospitalares para desenvolver ações educativas. Os temas abordados são apresentados por graduandos, de forma lúdica, simples, com figuras ilustrativas ou jogos motivadores de discussão, estimulando a troca de experiências entre pacientes, acadêmicos e profissionais de saúde. Também foram realizadas três oficinas culinárias e envio de 37 mensagens, por aplicativo, relembrando o conteúdo discutido presencialmente e mantendo vínculo entre paciente e equipe de saúde. Os resultados das ações educativas são avaliados por testes e demonstram que, em 14 ações, os acertos no pós-teste superaram os do préteste. Concluiu-se que o projeto contribui para a promoção da saúde dos pacientes e para a formação profissional dos discentes.
\end{abstract}

Palavras-chave: Diabetes mellitus tipo 2. Doenças crônicas. Idoso. Promoção da saúde.

Abstract: Diabetes education is an ongoing process of access to knowledge for patients to develop skills for disease management. With this in mind, the present project occupies the time of waiting for hospital consultations to develop educational actions. The topics covered are presented by graduates, in a playful, simple way,

\footnotetext{
1 Bolsista do PROBEC-UFG 2017/2018

2 Bolsista do PROBEC-UFG 2016/2017

3 Acadêmicas e participantes do PROVEC 2017/2018

4 Docentes da Faculdade de Nutrição UFG
} 
with illustrative figures or games motivating discussion, stimulating the exchange of experiences among patients, academics and health professionals. Three culinary workshops were also held and 37 messages were sent by mobile app, recalling the content discussed in person and maintaining a link between the patient and the health professionals. The results of the educational actions are evaluated by tests and demonstrate that, in 14 actions, the correct answers in the post-test exceeded those in the pre-test. It was concluded that the project contributes to the promotion of the patients' health and to the academic background of undergraduates.

Keywords: Type 2 diabetes mellitus. Chronic diseases. Elderly. Health promotion.

Resumen: La educación en diabetes es un proceso continuo de acceso al conocimiento para que los pacientes desarrollen habilidades para el manejo de la enfermedad. Pensando así, el presente proyecto ocupa el tiempo de espera por consultas hospitalarias para desarrollar acciones educativas. Los temas abordados son presentados por graduandos, de forma lúdica, simple, con figuras ilustrativas o juegos motivadores de discusión, estimulando el intercambio de experiencias entre pacientes, académicos y profesionales de salud. También se realizaron tres talleres culinarios y envío de 37 mensajes, por aplicación, recordando el contenido discutido presencialmente y manteniendo vínculo entre paciente y equipo de salud. Los resultados de las acciones educativas son evaluados por pruebas y demuestran que, en 14 acciones, los aciertos en el post-test superaron los del pre-test. Se concluyó que el proyecto contribuye a la promoción de la salud de los pacientes ya la formación profesional de los alumnos.

Palabras clave: Diabetes mellitus tipo 2. Enfermedades crónicas. Ancianos. Promoción de la salud.

\section{INTRODUÇÃO}

O Diabetes mellitus (DM) é um distúrbio metabólico caracterizado pela hiperglicemia crônica, resultante de defeitos na ação e/ou na secreção da insulina, ocasionando complicações em longo prazo. A manutenção crônica da glicemia elevada poderá resultar em distúrbios micro e macrovasculares, aumento de morbidade, redução da qualidade de vida e elevação da taxa de mortalidade (OLIVEIRA; MONTENEGRO JUNIOR; VENCIO, 2017).

A classificação do DM proposta pela Organização Mundial da Saúde (OMS) e pela Associação Americana de Diabetes (ADA) inclui quatro classes clínicas: DM tipo 1 (DM1), DM tipo 2 (DM2), outros tipos específicos de DM 
e DM gestacional (ALBERTI; ZIMMET, 1999; ADA, 2014-15). O DM1 é uma doença autoimune, poligênica, decorrente de destruição das células $\beta$ pancreáticas, ocasionando deficiência insulínica completa. Já o DM2 apresenta etiologia complexa e multifatorial, envolvendo componentes genéticos, ainda não completamente esclarecidos, e ambientais. Dentre os principais fatores de risco modificáveis para o DM2 encontram-se os hábitos dietéticos e a inatividade física, que contribuem para a obesidade com consequente resistência à insulina. Outros tipos de DM podem estar relacionados a defeitos ou síndromes genéticas, doenças do pâncreas exócrino, secundário às infecções ou ao uso de medicamentos, entre outros. Por fim, o DM gestacional é uma intolerância a carboidratos, geralmente diagnosticada no segundo ou terceiro trimestre da gestação. O DM gestacional pode ser transitório ou persistir após o parto, sendo importante fator de risco para desenvolvimento de DM2 (OLIVEIRA; MONTENEGRO JUNIOR; VENCIO, 2017).

Estima-se que, atualmente, a população mundial com diabetes seja de 415 milhões de pessoas, com $75 \%$ dos pacientes residindo em países em desenvolvimento, e que alcance 642 milhões em 2040. No Brasil, a prevalência de diabetes é de 10 a $20 \%$ na faixa etária de 20 a 79 anos, e o país ocupa a quarta posição na classificação mundial pelo número de pessoas acometidas pela doença (IDF, 2015). O número de indivíduos com diabetes está aumentando em virtude do crescimento e do envelhecimento populacional, da maior urbanização, do progressivo aumento da população sedentária e com obesidade e da maior sobrevida de pacientes com diagnóstico de diabetes (SBD, 2015-16).

Quanto à mortalidade, atribui-se ao diabetes 5,2\% de todos os óbitos no mundo, o que faz esta doença a quinta principal causa de morte (ROGLIC; UNWIN; BENNETT et al., 2005). A doença cardiovascular é a principal causa de óbito entre indivíduos com diabetes e representa metade dos falecimentos por diabetes, na maioria dos países, mesmo com subestimativa das estatísticas (OLIVEIRA; MONTENEGRO JUNIOR; VENCIO, 2017). Estudos no Brasil, quando se menciona DM na declaração de óbito, mostram que a taxa de mortalidade por essa enfermidade aumenta até 6,4 vezes (FRANCO, 2004) e que uma parcela importante desses óbitos ocorre de forma prematura, quando ainda os indivíduos contribuem economicamente para a sociedade (ROGLIC; UNWIN; BENNETT et al., 2005).

Deve-se considerar que a natureza crônica do DM, a gravidade das complicações e os meios necessários para controle tornam o DM uma doença muito 
onerosa para os indivíduos afetados e suas famílias e para o sistema de saúde (WHO, 2002). Estimativas do custo direto anual do DM, para o Brasil, oscilam em torno de 3,9 bilhões de dólares. No tratamento ambulatorial pelo Sistema Único de Saúde (SUS) brasileiro, os custos são da ordem de US\$2.108,00 por paciente, dos quais US\$1.335,00 estão relacionados a custos diretos (BAHIA et al., 2011). Além dos custos econômicos temos os custos intangíveis, como a dor, a ansiedade, a dependência de outras pessoas para atividades rotineiras, acarretando a perda de qualidade de vida das pessoas com diabetes e seus familiares (OLIVEIRA; MONTENEGRO JUNIOR; VENCIO, 2017).

O controle metabólico do indivíduo com DM, identificado pela hemoglobina glicada (HbAlc) entre 6,5 e $7 \%$, é a principal meta para a prevenção de complicações e das doenças associadas ao diabetes. No entanto o tratamento do diabetes vem se tornando cada vez mais complexo com a incorporação de novas tecnologias ao alcance do paciente para seu uso diário. Os profissionais de saúde e os pacientes são instigados a lidar com inúmeras informações e necessitam compartilhar conhecimentos para melhor controle da doença (OLIVEIRA; MONTENEGRO JUNIOR; VENCIO, 2017).

O sucesso no controle do diabetes necessita do estabelecimento e do desenvolvimento de integração entre órgãos governamentais e sociedade civil, restabelecendo-se corresponsabilidade em ações orientadas para prevenção, detecção e controle da doença (FERNANDES et al., 2016). Desde o início do diagnóstico, pacientes e seus familiares devem procurar adquirir os conhecimentos sobre a doença, comorbidades e cuidado nutricional e desenvolver habilidades necessárias para o autocuidado. A educação em diabetes é o processo contínuo de facilitação e acesso ao conhecimento e deve promover o desenvolvimento das habilidades necessárias para o gerenciamento da doença pelo próprio paciente e/ou familiar/cuidador (TORRES; PEREIRA; ALEXANDRE, 2011). Entretanto, observou-se que, no Brasil, um quinto dos portadores de diabetes não realiza tratamento adequado, mostrando que ainda se tem muito a avançar em relação ao cuidado destes indivíduos (ORTIZ; ZANETTI, 2001).

Portanto, é fundamental o desenvolvimento de ações de educação em saúde, tanto individuais quanto coletivas, com o objetivo de promover a melhora dos resultados clínicos, da qualidade de vida do paciente e alcançar a prevenção de complicações crônicas (SALCI; MEIRELLES; SILVA, 2018). Os espações de saúde, sejam do nível primário ao terciário, devem ser de acolhimento e transformação do paciente como cidadão, de forma humanizada, 
tornando-o corresponsável pelo seu tratamento. Neste sentido, o objetivo geral deste trabalho foi desenvolver ações educativas relacionadas com nutrição no diabetes mellitus tipo 2, auxiliando o paciente e sua família, usuários do SUS, nos cuidados exigidos pela doença. Também foram objetivos do projeto possibilitar aos acadêmicos participar de atividades de extensão universitária e aprofundar os conhecimentos sobre a temática em questão.

\section{Metodologia}

\section{Projeto de extensão}

Trata-se de um projeto de extensão da Faculdade de Nutrição da Universidade Federal de Goiás (UFG) iniciado em 2016 e ainda em andamento, cadastrado na Pró-Reitoria de Extensão e Cultura da UFG. As ações educativas são realizadas no Hospital das Clínicas (HC/UFG) de Goiás para pacientes com DM2 em espera de atendimento ambulatorial médico e/ou nutricional.

\section{Seleção dos alunos participantes do projeto}

A equipe de trabalho foi selecionada, num primeiro momento, a partir da divulgação junto aos acadêmicos dos referidos cursos da saúde da UFG e, posteriormente, por meio do Sistema Integrado de Gestão de Atividades Acadêmicas (SIGAA)/Extensão tendo como critério fundamental o interesse, a motivação e a disponibilidade de horário para desenvolver a atividade. Atualmente o projeto conta com uma bolsista do Programa de Bolsas de Extensão e Cultura (PROBEC/UFG) e seis alunas do Programa de Voluntários de Extensão e Cultura (PROVEC/UFG).

\section{Capacitação dos discentes}

A capacitação dos alunos foi realizada por meio da apresentação da proposta de trabalho e construção coletiva do plano de trabalho na seguinte sequência:

- Plano de ação: composto pelo levantamento de demandas dos pacientes, trazidas pelos residentes da área médica e multiprofissional do $\mathrm{HC} /$ UFG e por estagiários da área de Nutrição Clínica do $9^{\circ}$ e $10^{\circ}$ períodos do curso de Nutrição da UFG. 
- Formação da equipe: inicialmente formado pelas áreas da saúde (nutrição, enfermagem e medicina) com futura possibilidade de participação de outras especialidades, como psicologia e educação física.

- Reuniões acadêmicas: são realizadas semanalmente ou de acordo com a necessidade, para discussão dos temas que serão abordados nas atividades e para avaliação das ações realizadas. Os alunos são orientados para um estudo bibliográfico sobre a saúde e qualidade de vida, conhecimentos e habilidades básicas para prevenção de doenças crônicas e seus agravos e de como adquirir recursos psicológicos e antropológicos para lidar com grupos humanos.

\section{Desenvolvimento das Atividades}

Depois de estabelecido um cronograma mensal de ações educativas, as atividades ocorrem semanalmente, às quintas-feiras, no período vespertino, e possuem duração de 30 minutos cada, podendo ocorrer, no máximo, duas ações no mesmo dia com distintos grupos. Os temas abordados são preparados e apresentados por acadêmicos da graduação, de forma lúdica, expressos em linguagem simples e com apresentação de figuras ilustrativas ou jogos motivadores de discussão, estimulando a participação dos indivíduos e a troca de experiências entre pacientes e acompanhantes, acadêmicos e profissionais de saúde. Os materiais educativos para cada ação são desenvolvidos pelas alunas participantes do projeto com orientação da professora responsável e discutem temas relacionados com o diabetes, alimentação e nutrição e dificuldades da rotina diária enfrentada pelos pacientes.

Ao iniciar a atividade do dia, aplica-se um pré-teste composto por três a quatro perguntas de múltipla escolha, como instrumento de avaliação, com objetivo de verificar o conhecimento prévio dos pacientes a respeito do tema que será abordado no dia. Após a realização da ação é aplicado um pós-teste (com as mesmas questões do pré-teste) para verificar a consolidação das informações e esclarecer as dúvidas que ainda permanecerem. Por fim é distribuído um teste em escala hedônica facial com cinco pontos, com objetivo de avaliar o quanto a atividade foi proveitosa para os participantes.

\section{Atividades extras à sala de espera}

Oficinas culinárias foram desenvolvidas, em comemoração ao Dia Internacional e Nacional do Diabetes. Essas atividades foram realizadas às sextas-feiras 
à tarde no laboratório de Técnica Dietética na Faculdade de Nutrição da Universidade Federal de Goiás no qual os pacientes preparam receitas salgadas e doces (sem adição de açúcar), saborosas e saudáveis, sob a supervisão das discentes. Durante as oficinas, os participantes também são orientados quanto às normas básicas de higiene, manuseio e conservação dos alimentos. Ao final há apresentação das preparações, degustação, compartilhamento de experiências e troca de ideias com sugestões e críticas sobre as receitas elaboradas. A equipe do projeto tem o cuidado de selecionar receitas adequadas ao nível socioeconômico e cultural dos participantes e se propôs, inclusive, a apresentar alimentos que não fazem parte do hábito alimentar do grupo, mas que são benéficos ao controle da glicemia, como alimentos ricos em fibras.

Outra atividade realizada é o envio semanal de mensagens de texto aos pacientes ou familiares/cuidadores, via celular, por meio de aplicativo de mensagem, utilizando-se uma via de transmissão. Considerando-se que o celular pode ser uma ferramenta poderosa para compartilhar conhecimento, frases curtas ou ilustrações relembrando o conteúdo discutido no encontro presencial, reforçam e completam os temas abordados, além de manter um vínculo importante entre o paciente e a equipe de saúde até as consultas de retorno.

\section{Análise estatística}

Para avaliar a distribuição das respostas corretas no pré e pós-teste utilizou-se teste $\mathrm{W}$ de Shapiro Wilk. Para cada variável foram obtidas estimativas para a média, em porcentagem. As comparações entre os acertos no pré e pós-teste foram realizadas pela utilização do teste t-pareado. O nível de significância adotado foi de $5 \%(\mathrm{p}<0,05)$.

\section{Resultados}

De agosto de 2016 a fevereiro de 2018, 175 pacientes e mais alguns acompanhantes não computados participaram das ações educativas em nutrição em diabetes, ministradas pelas acadêmicas integrantes deste projeto de extensão, com a supervisão da coordenadora do projeto. As ações desenvolvidas e executadas durante a sala de espera abordaram os seguintes temas: nutrientes componentes dos alimentos; alimentação saudável; diferenças entre as terminologias diet, light e zero; entendendo os rótulos dos alimentos industrializados; interações entre medicamentos e alimentos; o que são hipoglicemias 
e hiperglicemias e seus sintomas; o que são receitas saudáveis e trocas de receitas entre os pacientes e discussão sobre as complicações do diabetes. Para a discussão foram elaborados pirâmide alimentar, adesivos com figuras de alimentos, pôsteres, quadros, jogos de perguntas e respostas, jogos como o "semáforo alimentar" e com rótulos de alimentos industrializados.

Os resultados obtidos nos pré e pós-teste mostraram que houve aumento do nível de conhecimento sobre os temas abordados, com exceção da primeira atividade, em agosto de 2016, talvez pela inexperiência inicial das discentes na condução do tema proposto ao grupo. Em média os pacientes acertaram $70,5 \%$ das questões do pré-teste e, após discussão, acertaram 88,5\%. Houve diferença significativa $(\mathrm{p}<0,05)$ em três momentos nos quais foram discutidos os rótulos dos alimentos industrializados, as interações entre medicamentos e alimentos e o que são hipoglicemias e hiperglicemias e seus sintomas. Observa-se que em oito ações houve um percentual de acerto no pós-teste acima de 96\% indicando concretização do tema abordado. Na Figura 1 encontram-se os percentuais de acertos obtidos nos pré e pós-teste aplicados mensalmente.

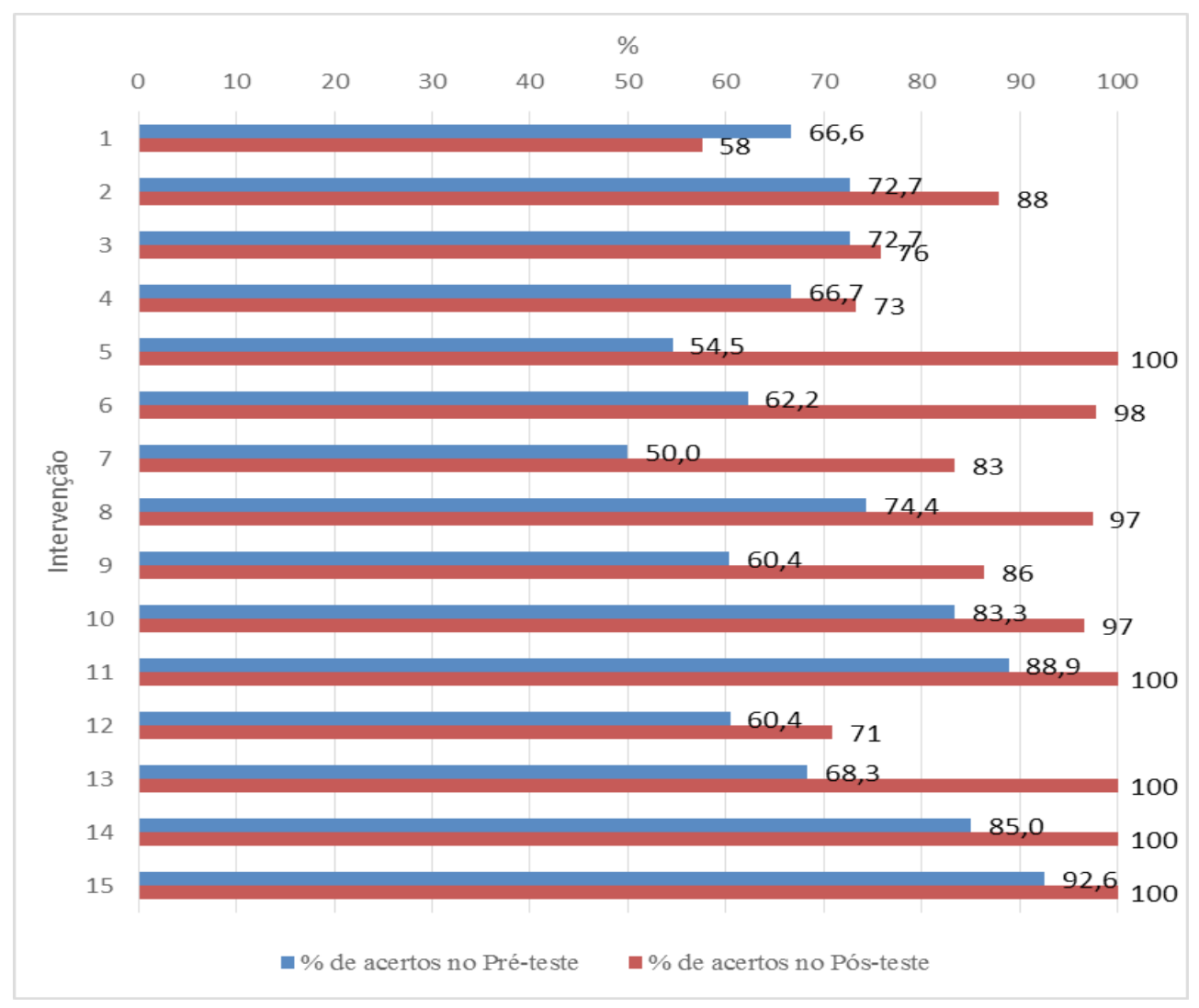

Figura 1. Percentual de acertos no pré e pós-teste aplicados nas intervenções mensais. 
Após a análise do teste em escala hedônica facial com cinco pontos, para a avaliação final da atividade pelos participantes, observa-se que $99 \%$ dos pacientes marcaram entre "adorei" e "gostei" demonstrando que as atividades propostas foram bem recebidas pelos pacientes (Figura 2).

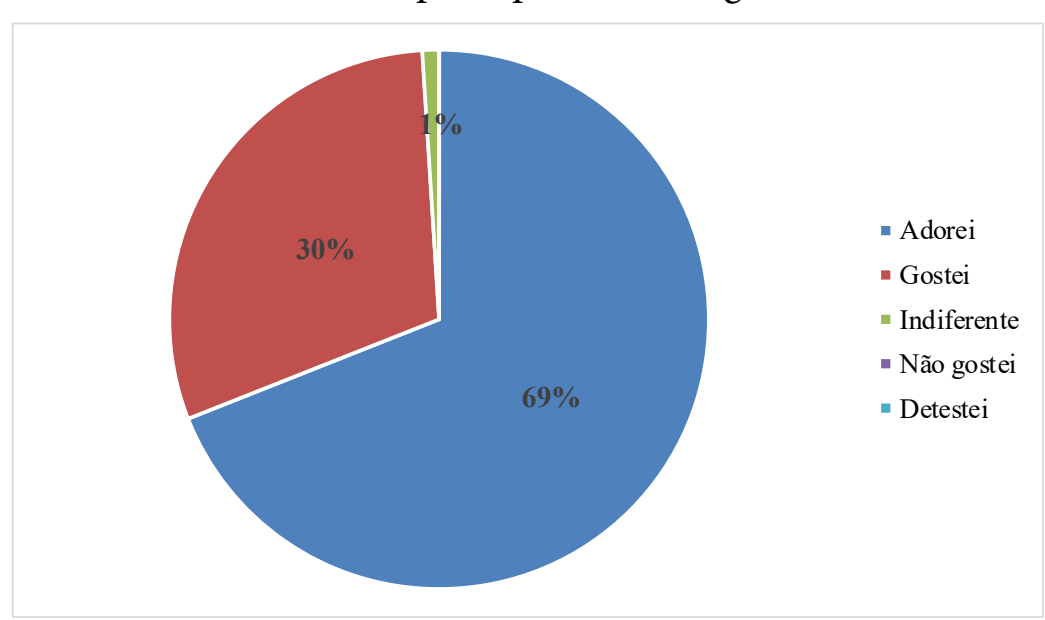

Figura 2. Avaliação da satisfação em participar da atividade.

Em comemoração ao Dia Internacional do Diabetes, em Novembro de 2016 e 2017, e Dia Nacional do Diabetes, em Junho de 2017, realizaram-se três oficinas culinárias das quais participaram, no total, 16 pacientes com diabetes, dois acompanhantes, discentes bolsistas e voluntárias, auxiliar de laboratório e professoras colaboradoras do projeto. Durante as oficinas, além de orientar quanto à higiene, manuseio e conservação dos alimentos e utensílios, foram elaboradas 28 tipos de preparações culinárias, sendo 15 salgadas e 13 doces, sem adição de açúcar (Quadro 1).

Quadro 1. Preparações culinárias desenvolvidas durante as oficinas.

\begin{tabular}{|c|c|}
\hline Preparações salgadas & Preparações doces (sem adição de açúcar) \\
\hline Arroz integral com carne seca & Bolo integral de maçã e de aveia com banana \\
\hline Arroz integral com brócolis & Cookies de cacau e aveia \\
\hline Arroz integral com frango & Brigadeiro \\
Lasanha de berinjela & Torta de limão \\
\hline Salada de macarrão integral & Arroz-doce \\
\hline Quibe de forno & Curau de milho \\
\hline Quibe de legumes ao forno & Pudim de baunilha \\
\hline Sanduiche natural de frango com cenoura & Paçoquinha \\
\hline Patê de cenoura com batata & Manjar de coco \\
\hline Patê de berinjela & Suco de morango, laranja e beterraba e suco \\
\end{tabular}


Ao final das oficinas culinárias, os participantes compartilharam dificuldades e facilidades sobre o preparo das receitas, degustaram os pratos preparados e receberam receituários com todas as preparações desenvolvidas no dia. Os pacientes e acompanhantes avaliaram positivamente a atividade uma vez que declararam que as receitas foram variadas e que as fariam, em seus domicílios, para seus familiares. Relataram também que o ambiente foi agradável e acolhedor e que aprenderam muito sobre alimentação saudável. A Figura 3 mostra as preparações elaboradas em uma das oficinas e parte da equipe do projeto.

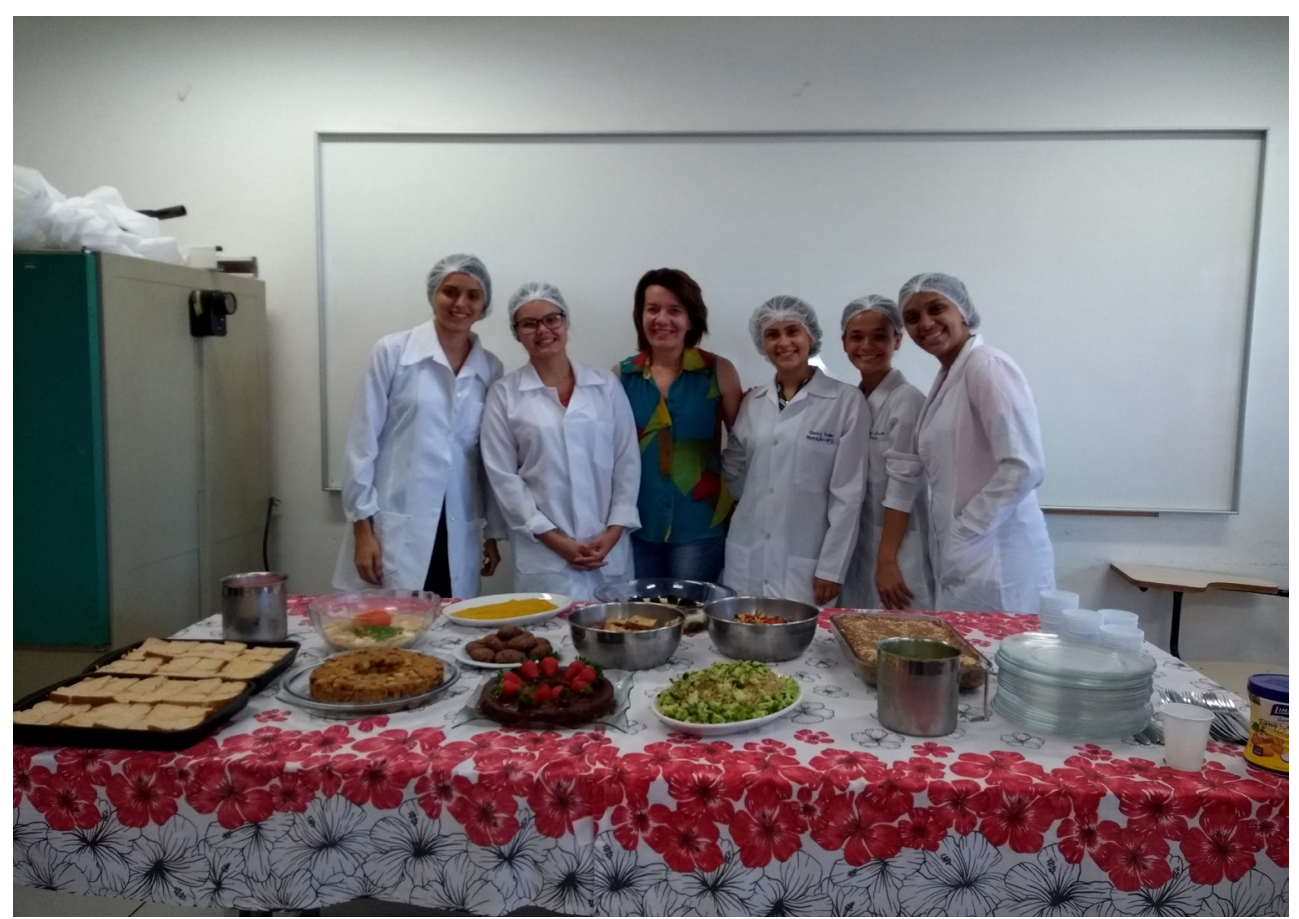

Figura 3. Equipe e preparações da oficina culinária para pacientes com diabetes.

Quanto ao envio semanal de mensagens de texto via aplicativo de celular foram transmitidas, no período de agosto de 2016 até fevereiro de 2018, 37 mensagens para 56 pacientes que possuíam aparelho telefônico móvel e acesso ao aplicativo de mensagens. Alguns temas das mensagens enviadas foram sobre a importância da alimentação saudável, do controle de peso ideal, da ingestão hídrica, do consumo de frutas e vegetais, de realizar atividade física orientada, dos cuidados com o pé diabético, observar os principais sintomas de hipoglicemia, conhecer as medidas de prevenção do diabetes tipo 2, como evitar o consumo exagerado de sódio, importância não fumar, entre outras. A utilização dessa ferramenta possibilitou reforçar e complementar informações 
sobre nutrição e diabetes, criando-se um vínculo entre o serviço de saúde e a comunidade. A Figura 4 mostra um exemplo de mensagem transmitida.

\section{Beneficios da fibra alimentar:}

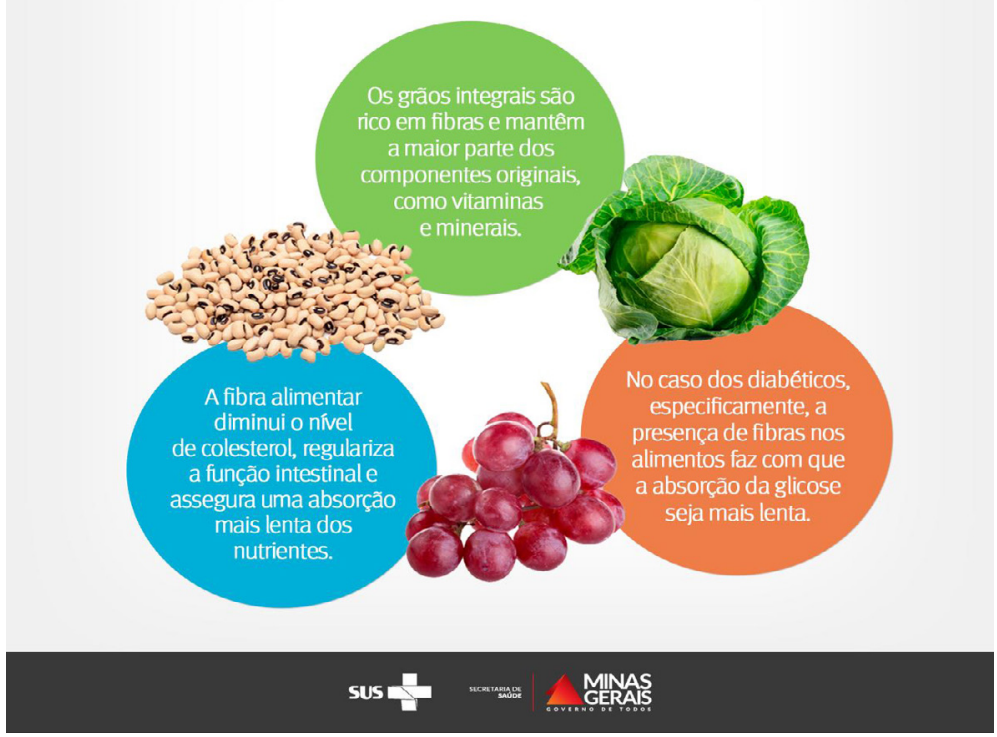

Figura 4. Exemplo de mensagem enviada aos pacientes com diabetes via aplicativo.

\section{Discussão}

Em 1978, a Conferência Internacional sobre Cuidados Primários de Saúde, ocorrida em Alma-Ata estabeleceu, como direito fundamental, a necessidade de promover a saúde de todos os indivíduos. Nesse contexto, a saúde passou a ter a dimensão de qualidade de vida e não simplesmente de ausência de doença, o que exige pessoas informadas sobre os cuidados em saúde e com capacidade para melhorar as condições físicas e psicossociais nos espaços em que vivem (Declaração de Alma-Ata, 1978).

Nesta perspectiva, novos serviços em saúde devem ser criados para atender as necessidades da população, fornecendo formas de acolhimento, humanizando a assistência e melhorando a qualidade dos serviços prestados a comunidade. Por meio de ações de "sala de espera" os profissionais da área da saúde têm a oportunidade de desenvolver atividades que extrapolam o cuidado, como a educação em saúde, melhorando a inter-relação usuário/sistema/ trabalhador de saúde (RODRIGUES et al., 2009). 
A sala de espera é o lugar onde os pacientes transitam e aguardam o atendimento dos profissionais de saúde. Por constituir em um espaço público, as pessoas, mesmo sem se conhecerem, conversam, trocam experiências entre si, observam, emocionam-se e expressam-se, ou seja, as pluralidades emergem através do processo interativo, mas sem criação de vínculo. Entretanto, quando alguma atividade se instala na sala de espera pela iniciativa dos profissionais de saúde, comumente, forma-se um trabalho de grupo, com um contexto definido, e ocorre, então, uma troca de experiências comuns, do saber popular e das distintas maneiras de cuidados com o corpo, de modo que o linguajar popular interage com os saberes dos profissionais de saúde (TEIXEIRA; VELOSO, 2006).

$\mathrm{Na}$ sala de espera observa-se que os participantes ficam à vontade para perguntar e esclarecer suas dúvidas, diferente no atendimento individualizado do ambulatório em que a relação com o profissional de saúde, às vezes, é mais tímida. Essa interação foi verificada entre os pacientes com diabetes e a equipe de nutrição durante e após as ações do presente projeto, principalmente entre os participantes das oficinas de culinária e também foi corroborado por Urbano et al. (2015) no sala de espera do serviço de Onco-hematologia Pediátrica do Instituto da Criança da Universidade de São Paulo.

A ação da sala de espera inicia-se com perguntas motivadoras para começar o processo interativo, de modo que os alunos questionam os participantes sobre suas sensações, percepções, dificuldades em suas vivências cotidianas e dúvidas sobre nutrição e o diabetes para enriquecer o processo de ensino-aprendizagem. Sendo assim, os conteúdos são transmitidos, em linguagem simples, e de modo dialógico com os usuários, favorecendo o retorno desses conteúdos assimilados, por meio dos pós-testes, de forma reflexiva. Nesta perspectiva, segundo Rodrigues et al. (2008), para que as ações desenvolvidas na sala de espera sejam efetivas é necessário utilizar tanto a linguagem verbal quanto a não verbal, uma vez que esta, ao abranger o toque, o olhar, os gestos, permite perceber e conhecer mais humanamente o outro, seus sentimentos, anseios, garantindo a melhor compreensão entre os usuários envolvidos, portanto, facilitando a comunicação.

A educação é uma estratégia fundamental para a promoção da saúde, visando atuar sobre o conhecimento das pessoas, para que elas desenvolvam a capacidade de intervenção sobre suas vidas e sobre o ambiente, criando condições para sua própria existência. As maiores pretensões das ações em prol da promoção à saúde são maximizar as possibilidades de evitar os agravos que 
as doenças trazem e de constituir indivíduos conscientes, responsáveis e com qualidade de vida (ROSA; BARTH; GERMANI, 2011)

Acredita-se que, para o sucesso da educação dos pacientes com diabetes, é imprescindível considerar os aspectos motivacionais para o autocuidado, a participação da família e o estabelecimento de vínculos afetivos com a equipe multiprofissional (FERRAZ et al., 2000). De acordo com Japur e Borges (2008), é necessário abrir espaços de diálogo com a população, gerando espaços de reflexão e problematização, como a sala de espera, que possibilitem a construção de uma relação de corresponsabilidade no tratamento.

Considerando-se a especificidade do diabetes como doença crônica e o controle glicêmico como fundamental na prevenção de complicações e sequelas, o conhecimento da doença por meio de educação é muito relevante no tratamento (PONTE et al., 2006). Mesmo que não se disponha de dados sobre o impacto deste projeto de sala de espera sobre o perfil clínico-metabólico dos pacientes com diabetes, percebe-se maior motivação dos pacientes após cada reunião, participação mais ativa nas consultas médicas e um crescente interesse sobre a sua enfermidade e o seu cuidado. Trabalho realizado por Torres et al. (2018) com 470 pessoas com diabetes mellitus tipo 2, que participaram de programa educativos no município de Belo Horizonte, verificou que as ações realizadas contribuíram para a manutenção do controle glicêmico, e até mesmo, redução da glicemia dos participantes.

Outra atividade a ser discutida são as oficinas culinárias onde se propõe uma experiência de vivência e reflexão sobre as relações entre alimentação, cultura e saúde, além de valorizar, no cotidiano, a prática de cozinhar. A falta de informação sobre o valor nutricional dos alimentos, aliado ao desconhecimento de seu preparo e aproveitamento máximo, são fatores que contribuem para o seu desperdício e monotonia de cardápios. As oficinas constituíram um espaço ideal para a apreensão de conhecimentos, informações e troca de experiências no que se refere aos benefícios de certos alimentos e sua inclusão na alimentação diária para o controle do DM e aos possíveis danos que outros podem causar ao organismo humano, quando esses são consumidos de forma incorreta (FIGUEIREDO et al., 2014).

Neste projeto de extensão também foram utilizadas tecnologias de informação e comunicação (TIC) para proporcionar continuidade ao processo de educação em saúde além das reuniões da sala de espera. O uso destas tecnologias se estabeleceu como uma das principais fontes de informação em saúde, 
no entanto, as estratégias que usam as TIC para auxiliar os indivíduos na mudança de comportamentos de saúde ainda são pouco exploradas (CURIONI; BRITO; BOCCOLINI, 2013). O envio de mensagens por aplicativo tem se mostrado efetiva para a mudança em curto prazo de comportamentos em saúde, como o autocuidado em diabetes, além de fornecer uma oportunidade singular para a promoção de estilos de vida saudáveis e atingir simultaneamente vários indivíduos (FJELDSOE; MARSHALL; MILLER, 2009). Contudo, as limitações no emprego das TIC em nutrição devem ser levadas em consideração, uma vez que dependem do acesso dos beneficiários a ferramentas de comunicação, da motivação e aptidão para seu manuseio e dos custos de sua execução (CURIONI; BRITO; BOCCOLINI, 2013).

Por fim, o projeto permite, aos acadêmicos, conhecimentos metodológicos acerca da extensão universitária e o aprofundamento dos conhecimentos sobre a temática em questão. A sala de espera favorece o contato com a realidade dos usuários do SUS, que é diversificada, envolvida por dimensões econômicas, sociais e pluriculturais. Tal experiência proporciona aos alunos o desenvolvimento de habilidade de se relacionar com pessoas, bem como manejar a própria capacidade emotiva e discursiva no processo de comunicação nas práticas educativas.

O ensino-aprendizagem permite formar profissionais críticos e reflexivos, capazes de construir novos conhecimentos a partir da realidade observada ao seu redor, tornando-os indivíduos com senso ético e humano. A extensão universitária contribui com a formação acadêmica dos alunos participantes dos projetos, fornecendo oportunidade para o estreitamento de laços com a sociedade e proporcionando condições para que o conhecimento transforme-se em sabedoria (SANTOS et al., 2011).

\section{Conclusão}

Diante de todo o processo de desenvolvimento das atividades na sala de espera concluiu-se que o projeto contribui para a promoção da saúde, uma vez que discute temas relacionados com as dificuldades diárias destes pacientes. Estas ações humanizam o serviço de saúde, proporcionando atenção integral à saúde dos pacientes e criam um vínculo entre os acadêmicos, profissionais de saúde e a sociedade. 
Esta prática precisa ser estimulada para o empoderamento do conhecimento dos pacientes e seus familiares sobre a doença e seu cuidado. O projeto também permite que os alunos percebam os pacientes como pessoas, cidadãos que podem se transformar e contribuir para uma mudança social e não apenas como meros portadores de patologias. Além disto, ações como esta motivam os discentes a participar de atividades de educação em saúde, desenvolvendo sua capacidade de se comunicar com o público. No que diz respeito à nutrição, pode-se dizer ainda que este projeto apresenta um espaço para divulgarmos a profissão e mostrarmos a qualidade do trabalho do profissional nutricionista.

Por fim, uma das dificuldades encontradas para o desenvolvimento do projeto é a falta de financiamento para a confecção dos materiais utilizados nas ações educativas e para a aquisição dos gêneros alimentícios usados nas oficinas de culinária. Atualmente, acadêmicos e docentes participantes do projeto custeiam estes produtos, porém com recursos advindos de fontes financiadoras, estes materiais poderiam ser elaborados com materiais mais resistentes, sendo utilizados, por mais vezes, em outras atividades de educação em saúde. Outro problema encontrado foi o dia e horário da oficina de culinária (sexta-feira à tarde) o que impedia a liberação dos pacientes de seus serviços para comparecerem à Faculdade de Nutrição. Assim, para facilitar a participação nesta oficina, alteramos a data para sábado pela manhã a partir de 2018. Quanto ao uso de TIC, embora a maioria dos participantes possua aparelho telefônico móvel, há dificuldade de acesso ao aplicativo e falha no manuseio. Apesar destes obstáculos, o projeto foi recadastrado no SIGAA/ extensão e continuará em 2018-19.

\section{REFERÊNCIAS}

ALBERTI, K.G.M.M.; ZIMMET, P.Z. World Health Organization Consultation. Definition, diagnosis and classification of diabetes mellitus and its complications. Part 1 : diagnosis and classification of diabetes mellitus. Report of a WHO Consultation. Geneva: WHO, 1999.

AMERICAN DIABETES ASSOCIATION. Diagnosis and classification of diabetes mellitus. Diabetes Care, Suppl 1, p. s81-90, 2014

BAHIA, L.R.; ARAUJO, D.V.; SCHAAN, B.D. et al. The costs of type 2 diabetes mellitus outpatient care in the Brazilian Public Health System. Value in Health, v.14, p. s137-40, 2011. 
CURIONI, C.C.; BRITO, F.S.B.; BOCCOLINI, C.S. O uso de tecnologias de informação e comunicação na área da nutrição. Jornal Brasileiro de Telessaúde, v. 2, n. 3, p. 103-11, 2013.

DECLARAÇÃO DE ALMA-ATA. Conferência Internacional sobre cuidados primários de saúde; 6-12 de setembro 1978; Alma-Ata; USSR. Disponível em: https://www. cmdss2011.org/site/wp-content/uploads/2011/07/Declaração-Alma-Ata.pdf. Acesso em 16 de fevereiro de 2018.

FERNANDES, J.R.; OGURTSOVA, K.; LINNENKAMP, U.; GUARIGUATA, L.; SEURING, T.; ZHANG, P. et al. IDF Diabetes Atlas estimates of 2014 global health expenditures on diabetes. Diabetes Research and Clinical Practice, n. 117, p. 48-54, 2016.

FERRAZ, A.E.P.; ZANETTI, M.L,; BRANDÃO, E.C.M.; ROMEU, L.C.; FOSS, M.C.; PACCOLA, G.M.G.F.; PAULA, F.J.A.; GOUVEIA, L.M.F.B.; MONTENEGRO Jr., R. M. Multiprofessional care of patients with diabetes mellitus at the Endocrinology Outpatient Clinic of the University Hospital of the Ribeirão Preto School of Medicine. (HCFMRP-USP). Medicina, v.33, p. 170-5, 2000.

FIGEIREDO, S.M.; FREITAS, M.C.D.; SILVA, J.M.T.; VIEIRA FILHO, S.A.; CALIGIORNE, R.B. Oficinas de culinária: método educativo de alimentação saudável. HU Revista, v. 40, n. 1 e 2, p. 69-74, 2014.

FJELDSOE, B.S.; MARSHALL, A.L.; MILLER, Y.D. Behavior change interventions delivered by mobile telephone short-message service. American Journal of Preventive Medicine, v.36, n. 2, p.165-73, 2009.

FRANCO, L.J. Um problema de saúde pública. Epidemiologia. In: Oliveira JEP, Milech A, eds. Diabetes mellitus: clínica, diagnóstico, tratamento multidisciplinar. São Paulo: Atheneu, 2004. p. 19-32.

INTERNATIONAL DIABETES FEDERATION. IDF Diabetes Atlas [Internet]. 7a ed. Brussels: International Diabetes Federation, 2015. Disponível em https://www.idf.org/e-library/epidemiology-research/diabetes-atlas/13-diabetes-atlas-seventh-edition. html. Acesso em 14 de fevereiro de 2018.

JAPUR, M.; BORGES, C. C. Sobre a (não) adesão ao tratamento: Ampliando sentidos do autocuidado. Texto e Contexto Enfermagem, v.17, n.1, p. 64-71, 2008.

OLIVEIRA, J. E. P.; MONTENEGRO JUNIOR, R. M.; VENCIO, S (Org.). Diretrizes da Sociedade Brasileira de Diabetes 2017-2018. São Paulo: Clannad, 2017. 398p.

ORTIZ, M.C.A.; ZANETTI, M.L. Levantamento dos fatores de risco para diabetes mellitus tipo 2 em uma instituição de ensino superior. Revista Latino-Americana de Enfermagem, Ribeirão Preto, v.9, n.3, p.58-63, 2001.

PONTE, C.M. M.; FERNANDES, V.O.; GURGEL, M.;COSTA, H. DA; VERAS, V.S.; QUIDUTE, A.R.P.; MONTENEGRO, R.M.; CARVALHO, S.L. de; MONTENEGRO JÚNIOR, R.M. Projeto sala de espera: uma proposta para a educação em diabetes. Revista Brasileira em Promoção de Saúde, v.19, n.4, p. 197-202, 2006. 
RODRIGUES, A.D.; NORA, C.R.D.; ROSA, J.; GERMANI, A.R.M. Sala de espera: um ambiente para efetivar a educação em saúde. Vivências, v.5, n.7, p.101-6, 2009.

RODRIGUES, A.D.; ROSA, J.; NORA, C. R. D.; GERMANI, A.R. Sala de espera: um ambiente de educação em saúde. Revista de Enfermagem (Frederico Westphalen), v. 4 e 5, n. 4 e 5, p. 61-73, 2008-2009.

ROGLIC, G.; UNWIN, N.; BENNETT, P.H. et al. The burden of mortality attributable to diabetes: realistic estimates for the year 2000. Diabetes Care, v.28, n.9, p.2130-5, 2005.

ROSA, J.; BARTH, P.O.; GERMANI, A.R.M. A sala de espera no agir em saúde: espaço de educação e promoção à saúde. Perspectiva, v.35, n.129, p. 121-30, 2011.

SALCI, M.A.; MEIRELLES, B.H.S.; SILVA, D.M.G.V. Educação em saúde para prevenção das complicações crônicas da diabetes mellitus na atenção primária. Escola Anna Nery Revista de Enfermagem, v. 22, n.1, p.1-6, 2018.

SANTOS, S.S.; SANTOS, N.A.; SOUZA, M.R..; BARCELOS, I.S.C. Educação em saúde na sala de espera: uma abordagem sobre amamentação. Em Extensão, v. 12, n. 1, p. 12934, 2013.

SOCIEDADE BRASILEIRA DE DIABETES. Epidemiologia e Prevenção. Disponível em http://www.sbd.org.br/. Acesso em 12 de fevereiro de 2018.

TEIXEIRA, E.R.; VELOSO, R.C. O grupo em sala de espera; território de práticas e representações em saúde. Texto e contexto- Enfermagem, v.15, n.2, p.320-5, 2006.

TORRES, H.C.; PACE, A.E.; CHAVES, F. F.; VELASQUEZ-MELENDEZ, G.; REIS, I.A. Avaliação dos efeitos de um programa educativo em diabetes: ensaio clínico randomizado. Revista de Saúde Pública, v.52, n.8, 2018.

TORRES, H.C.; PEREIRA, F.R.L; ALEXANDRE, L.R. Avaliação das ações educativas na promoção do autogerenciamento dos cuidados em diabetes mellitus tipo. Revista da Escola de Enfermagem USP, São Paulo, n.45, v.5, p.1077-1082, 2011.

URBANO, A.S.; MONTEIRO, R. M. da C.; CRISTÓFANI, L. M.; CURY, M.R.G.; NABARRETE, J.M.; ANDRADE, B.A. Sala de Espera: Uma Experiência no Serviço de Onco-Hematologia Pediátrica do Instituto da Criança do HCFMUSP. Disponível em http:// www.redehumanizasus.net/. Acesso em 20 de julho de 2018.

WORLD HEALTH ORGANIZATION. Diabetes: the cost of diabetes. WHO fact sheet. 2002. Sep, n. 236. 